\title{
Dihydropyridines as calcium channel blockers: an overview
}

\begin{abstract}
Cardiovascular disease remains one of the leading causes of death in the United States. It is estimated that 1 in every 3 American adults suffer from high blood pressure. Hypertension, a precursor to most cardiovascular diseases, continues to grow at an alarming rate and is seldom managed carefully. Attempts have been made to manage hypertension and reduce the morbidity and mortality of the cardiovascular organ through methods including diets, increased amount of exercise, and medications. Many commercial drugs have been derived from dihydropyridine due to its antihypertensive property. Dihydropyridine derivatives work by acting as calcium channel blockers blocking the intake of calcium ions into the vascular smooth muscle and, to a lesser extent, cardiac muscles. Dihydropyridine moiety is well-known in pharmacology as L-type calcium channel blockers, which is extremely important since it treats hypertension in people who suffer from it. It is also highly important to understand the antagonists of calcium in the arterial hypertension. A brief overview is presented in this mini-review.
\end{abstract}

Keywords: dihydropyridine, heterocycles, hypertension, cardiovascular disease, calcium channel
Volume 5 Issue 4 - 2017

\author{
Thelma Salazar,Andres Gonzalez, Debasish \\ Bandyopadhyay \\ Department of Chemistry, The University of Texas-Rio Grande \\ Valley, USA
}

\begin{abstract}
Correspondence: Debasish Bandyopadhyay, Department of Chemistry, The University of Texas-Rio Grande Valley, I20I West University Drive, Edinburg, Texas-78539, USA, Tel + I (956)57894 I 4, Fax + I(956)3845006

Email debasish.bandyopadhyay@utrgv.edu
\end{abstract}

Received: July 04, 2017 | Published: July 17, 2017

\section{Introduction}

Several heterocyclic compounds (both synthetic and natural) are widely known and many of them possess significant biological activities. ${ }^{1-5}$ In 2013, four out of top six and nine out of top twenty one highest selling drugs were small molecule heterocyclic compounds. ${ }^{6}$ Dihydropyridine is one of the important heterocyclic scaffolds that is frequently found in several medicines that include, but are not limited to, L-type calcium channel blocker (antihypertensive), ${ }^{7-9} \mathrm{~N}$-type channel blocker, ${ }^{10,11}$ antianginal, ${ }^{12}$ antithrombotic, ${ }^{13}$ antimineralocorticoid,,${ }^{14}$ anticonvulsant, ${ }^{15}$ anti-inflammatory, ${ }^{16,17}$ anticancer, ${ }^{18}$ and analgesic. ${ }^{19}$ On the other hand, high blood pressure, also known as hypertension, is a condition in where a higher than normal force pushes against the walls of the arteries as blood is pumped through them. It is estimated that 75million Americans, 1 in 3 adults, suffers from hypertension. Of that number, only $54 \%$ have their condition under control. Only in 2014, there were over 410,000 American deaths in which high blood pressure was the primary or contributing factor to the cause of death. ${ }^{20}$ This mini-review highlights the role of dihydropyridine derivatives as potential drugs to manage hypertension and the diseases associated with it.

\section{Discussion}

In order to a get a clear understanding about the role of dihydropyridine derivatives in human body it is important to know the role that L-type calcium channels as well as calcium channel blockers. A few dihydropyridine derived commercial drugs are presented in Figure 1.

\section{L-type calcium channels}

Calcium channels are part of plasma membrane proteins that are made up of calcium selective pores that are opened by a change in the voltage of the membrane. Calcium channels are found in neurons, vascular smooth muscles, cardiac muscles, and other excitable cells. Calcium channels are responsible for a variety of physiological functions such as neurotransmitter release, hormone release, muscle contraction, and vasodilation among other things.
Calcium channels play a role in a variety of diseases, including hypertension. ${ }^{21}$ The voltage- dependent calcium ion channels of the membrane are subdivided into four fundamental types according to their electrophysiology and sensitivity to certain drugs and toxins. These channels are called: Channels L, which has long activation and high conductance. They are mainly located in skeletal, cardiac and vascular muscle and its function is contraction. T-channels are transient opening which function is calcium ion input to negative levels of membrane potential. $\mathrm{N}$ channels which function is to release transmitters in cerebral synaptosomes. P channels which are localized in the cerebellum cells. Channels A whose characteristics are still being studied. The L-type, N-type and T-type voltage-dependent calcium channels are found in the zona glomerulosa in adrenal and the L-type calcium channels are a specialized type of voltage-gated calcium channel. Voltage-gated calcium channels selectively permeate calcium ions into the cell when there is a change from a high voltage to a low voltage inside of the cell. L-type calcium channels are the primary route for $\mathrm{Ca}^{2+}$ to enter the cardiac muscles. ${ }^{22}$

\section{Calcium channel blockers}

Calcium channel blockers (also known as calcium channel antagonists) are several medications that inhibit the movement of $\mathrm{Ca}^{2+}$ through calcium channels. There are three main classes of L-type channel blockers: phenylalkyamines, benzothiazapines, and 1,4-dihydropyridines. These approved calcium channel blockers bind to the L-type calcium channels in the cardiac tissue and smooth muscles. Since the function of the calcium channels is to regulate the amount of calcium that enters into the cell, the function of the calcium channel blockers is to prevent the transmembrane protein from allowing calcium into the cell.

When calcium is allowed into the vascular smooth muscle and cardiac muscles it causes smooth muscle contraction. People that suffer from high blood pressure do not want their arteries to contract because that increases the amount of pressure needed to circulate the blood in the body which results in an increased amount of strain placed on the heart and other cardiovascular organs. 


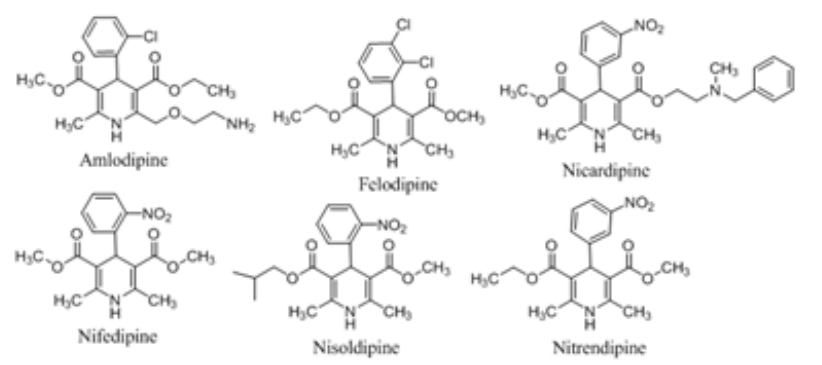

Figure I Example of a few dihydropyridine-containing antihypertensive commercial drugs.

Having calcium channel blockers inhibit the influx of calcium to the cell results in the relaxation of the vascular smooth muscle (vasodilation) and decreased systemic vascular resistance which lowers blood pressure and arterial pressure, which is a major contributor to chest pain (angina). ${ }^{23}$

\section{Mechanism of action}

The presence of calcium is essential for muscle to contract and according to the different reserve of this ion in the sarcoplasmic reticulum of the myofibrils, its absence is noticed with greater intensity in the smooth muscle than in the myocardium and the striated muscle. For this reason, the action of the calcium antagonists is greater on the smooth muscle of the walls. Although the entry of calcium ions is required for the release of neurotransmitters, calcium antagonists can directly activate the release of neurotransmitters through a mechanism that does not depend on a reflex response. The release takes place by the opening of storage granules. In this regard, the relative potency of the calcium antagonists is as follows: felodipine is greater than nicardipine and it is greater than amlodipine. The potential clinical relevance of these differences in potency should never be underestimated because there are many situations where the discharge of transmitters is far from desirable, such as in myocardial infraction and heart failure. The binding sites of calcium with antagonists are heterogeneous. Three sites of recognition of the dihydropyridines are known, which are accessed from the extracellular surface of the membrane. To some extent, the slow onset of the effects of amlodipine and in part its special pharmacological profile is a consequence of the ionization of the molecule at normal physiological $\mathrm{pH}$, which prevents its approach to the binding sites. The participation of calcium ions in smooth muscle contraction is a well-known phenomenon. Calcium ion can originate from two different sources: The extracellular calcium ion, which penetrates fundamentally through the L-type calcium ion channels and the calcium ion of the intracellular deposits that is released after receiving the corresponding signal. For example the neurotransmitter: norepinephrine.

Regardless of the source, calcium ion, known as calcium activator, binds to a regulatory protein, calmodulin that complexes with calcium and activates the enzyme protein kinase, which in turn facilitates the phosphorylation of myosin. The key event is the phosphorylation of myosin, which comes into contact with actin and contraction occurs. As the essential characteristic of hypertension is the increase in peripheral vascular resistance and since smooth muscle contraction depends on calcium ion, its antagonists are an effective treatment in this situation provided that: A considerable proportion of calcium ion, involved in events that culminate with contraction penetrating into the cytosol through the channels of calcium ion type L and counteract some of the other effects of hypertension, such as cardiac hypertrophy and atherosclerosis.
There are different reasons to argue that excess of calcium ion entry through the selective calcium ion L-type channels contributes to the increase in vascular resistance induced by arterial hypertension. So, intake of calcium ion excess occurs because the time is prolonged, resulting in an increase in intercellular deposits of exchangeable calcium ion. In the smooth muscle cells, the calcium ion tension concentration curve has a very steep slope, so that little calcium ion is required to trigger an excessive response. Increased arteriolar tone is counteracted by the addition of a calcium antagonist. It is admitted that the etiology of arterial hypertension is very complex and multifactorial, involving different factors such as: genetic predisposition of the disease; abnormal operation of the L-type calcium ion channels, which implies a relative increase in the number of functional channels as well as the opening time of each channel. Given all these elements, it would be very difficult to remove the calcium antagonists in the list of drugs potentially useful in hypertension, unless their side effects outweigh their beneficial actions. Calcium antagonists reduce systolic and diastolic blood pressure without causing arthrostatic or postural hypertension, without causing water and sodium retention, without altering the circadian pattern of blood pressure, without altering the lipid profile in the plasma, does not retain uric acid nor alter glycaemia. It maintains or improves renal blood flow and the decrease in blood pressure they cause does not always lead to a sustained increase in renin or angiotensin II. However, in the acute phases, plasma levels of noradrenaline may be increased due to a release of the reflex stimulated sympathetic neurotransmission in response to the sudden drop in peripheral vascular resistance and the direct stimulating effect of the release of noradrenaline from the storage granules.

The inherit limitations of these drugs are: its negative inotropic effect; in the case of nifedipine the rapidity of the fall in blood pressure, which causes reflex achycardia and stimulation of the sympathetic and renin-agiotensin systems. Prolonged-release preparations attempt to modify these unwanted responses; the depressant effect of verapamil, and to a lesser extent, diltiazem on the conduction system, determines sustained bradycardia in some cases. The reduced plasma half-life necessitates the administration of multiple doses, with the exception of amlodipine.

Therapeutic doses are usually associated with side effects such as constipation, bradycardia, edema. Hypertension is a vascular disease that is usually accompanied by a decrease, not an increase, in cardiac output and volume per heartbeat, so it is logical to choose a calcium antagonist with vascular selectivity that does not stimulate the release of catecholamine. The problem would be to know if they are still effective. At present, antagonists with vascular selectivity share two characteristics: they all are dihydropyridine derived, and as a consequence of their selectivity, are a logical alternative in the treatment of arterial hypertension. However, there are differences between the different selective vascular drugs, so amlodipine does not modify plasma noradrenalide, felodipine causes an increase that persists often in prolonged treatment. In the case of amlodipine, its hypotensive effect occurs without increasing the heart rate, whereas felodipine usually produces a reflex tachycardia. Amlodipine as a consequence of its prolonged plasma half-life and high bioavailability can be administered in a single dose per day; On the other hand, its action starts slowly and the possibility of producing reflex changes in the heart rate is quite remote, does not produce changes in plasma volume or sodium ion retention, nor does it present significant alterations in the logogram.

Antagonists with vascular selectivity reduce systemic pressure by decreasing peripheral vascular resistance and not by changes in cardiac output or volume depletion. Nifedipine may possibly meet 
the requirements of being a selective antagonist by exerting its best effect on the vessels compared to the myocardium, and also has no (or very little) effect on nodal tissue or atrioventricular conduction; The problem is that its selectivity for vessels is associated with rapid uptake and plasma distribution, as well as a rapid rate of fixation to its "receptor" in the complex of the calcium ion channel, for this reason produces rapid peripheral vasodilation. As a consequence, the autonomic nervous system is stimulated, determining a rapid and significant increase of the heart rate, which forces to concomitant treatment with $\beta$-blocker or a preparation of prolonged release. ${ }^{24-29}$

\section{Conclusion}

Clinical and laboratory observations of prototypes of calcium antagonists have revealed some interesting and possibly unexpected but clinically useful properties of these drugs. Thus, they are known to interfere with the processes leading to atherogenic development, to delay left ventricular hypertrophy induced by hypertension, to inhibit the growth of cancer cells, to improve the viability of the conserved organs, to protect against the destructive effect of free radicals and act as antiepileptics or potentiate the antimalarial effects of chloroquine. These properties, or at least some of them, should be maintained in the new generation of dihydropyrine-containing calcium antagonists. Amlodipine, nitrendipine, felodipine, nisoldipine prolong their halflife in patients with liver dysfunction. The antagonists are metabolized in the liver and therefore the dosage must be carefully adjusted in these cases.

\section{Acknowledgements}

The authors are thankful to the Department of Chemistry, College of Science, The University of Texas Rio Grande Valley.

\section{Conflicts of interest}

Author declares there are no conflicts of interest.

\section{Funding}

None.

\section{References}

1. Bandyopadhyay D, Rhodes E, Banik BK. A green, chemoselective, and practical approach toward $N$-(2-azetidinonyl) 2,5-disubstituted pyrroles. RSC Advances. 2013;3:16756-16764.

2. Bandyopadhyay D, Cruz J, Banik BK. Novel synthesis of 3-pyrrole substituted $\beta$-lactams via microwave-induced bismuth nitrate-catalyzed reaction. Tetrahedron. 2012;68:10686-10695.

3. Bandyopadhyay D, Mukherjee S, Banik BK. An expeditious synthesis of $\mathrm{N}$-substituted pyrroles via microwave-induced iodine-catalyzed reaction under solventless conditions. Molecules. 2010;15(4):2520-2525.

4. Rivera S, Bandyopadhyay D, Banik BK. Facile synthesis of $N$-substituted pyrroles via microwave-induced bismuth nitrate-catalyzed reaction under solventless conditions. Tetrahedron Letters. 2009;50:5445-5448.

5. Bandyopadhyay D, Mukherjee S, Granados JC, et al. Ultrasoundassisted bismuth nitrate-induced green synthesis of novel pyrrole derivatives and their biological evaluation as anticancer agents. Eur $J$ Med Chem. 2012;50:209-215.

6. http://www.drugs.com/stats/top100/2013/sales

7. Bandyopadhyay D, Maldonado S, Banik BK. Microwave-assisted bismuth nitrate-catalyzed unique route toward 1,4-dihydropyridines. Molecules. 2012;17(3):2643-2662.

8. Wang AL, Ladecola C, Wang G. New generations of dihydropyridines for treatment of hypertension. J Geriatr Cardiol. 2017;14(1):64-72.
9. Teleb M, Zhang FX, Farghaly AM, et al. Synthesis of new N3substituted dihydropyrimidine derivatives as L-/T- type calcium channel blockers. Eur J Med Chem. 2017;134:52-61.

10. Masaki M, Mano T, Eguchi A, et al. Long-term effects of L- and N-type calcium channel blocker on uric acid levels and left atrial volume in hypertensive patients. Heart Vessels. 2016;31(11):1826-1833.

11. Triggle DJ. The pharmacology of ion channels: with particular reference to voltagegated $\mathrm{Ca}+2$ channels. Eur J Pharmacology. $1999 ; 375(1-3): 311-325$.

12. Love B, Goodman M, Snader K, et al. Hantzsch-type dihydropyridine hypertensive agent. J Med Chem . 1974;17(9):956-965.

13. Sunkel CE, De casa JMF, Santos L. 4- alkyl-1,4-dihydropyridines as specific PAFacether antagonists. Journal of Medicinal Chemistry. 1990;33(12):3205-3210.

14. Kosaka H, Hirayama K, Yoda N, et al. The L-, N-, and T-type triple calcium channel blocker benidipine acts as an antagonist of mineralocorticoid receptor, a member of nuclear receptor family. Eur $J$ Pharmacol . 2010;635(1-3):49-55.

15. Tusell JM, Barron S, Seratosa J. Anticonvulsant activity of $-\mathrm{HCH}$, calcium channel blockers and calmodulin antagonists in seizures induced by lindane and other convulsant drug. Brain Res. 1993;622:99-104.

16. Briukhanov VM. The effect of $\mathrm{Ca}^{2+}$ antagonist on the development of inflammatory edema in rats. Exp Clin Pharmocol . 1994;57(2):47-49.

17. Bahekar S, Shinde D. Synthesis and anti-inflammatory activity of 1 , 4-dihydropyridines. Acta pharmaceutica (Zagreb) A. 2002;52:281-287.

18. Boer R, Gekeler V. Chemosensitiser in tumour therapy: new compounds promise better efficacy. Drugs Fut . 1995;20:499-509.

19. Gullapalli S, Ramarao P. L-type $\mathrm{Ca}^{2+}$ channel modulation by dihydropyridines potentiates- K-opioid receptor agonist induced acute analgesia and inhibits development of tolerance in rats. Neuropharmacology. 2002;42:467-475.

20. https://www.cdc.gov/dhdsp/data_statistics/fact_sheets/fs bloodpressure.htm

21. Bean BP, McDonough SI. Calcium Channels. John Wiley \& Sons Ltd, Chichester, England. 2010.

22. Snutch TP, Peloquin J, Mathews E, et al. Molecular Properties of Voltage-Gated Calcium Channels. Madame Curie Bioscience Database. 2017.

23. http://cvpharmacology.com/vasodilator/CCB

24. Tani S, Asayama K, Oiwa K, et al. The effects of increasing calcium channel blocker dose vs. adding a diuretic to treatment regimens for patients with uncontrolled hypertension. Hypertens Res. 2017.

25. Fan Z, Lv N, Luo X, et al. Isosteviol prevents the prolongation of action potential in hypertrophied cardiomyoctyes by regulating transient outward potassium and L-type calcium channels. Biochim Biophys Acta. 2017;1859(10):1872-1879.

26. Ahmad KA, Yuan YD, Nawaz W, et al. Antioxidant therapy for Management of Oxidative stress Induced Hypertension. Free Radic Res. 2017;51(4):428-443.

27. Cordeanu EM, Gaertner S, Faller A, et al. Rifampicin reverses nicardipine effect inducing uncontrolled essential hypertension. Fundam Clin Pharmacol. 2017.

28. Saddala MS, Kandimalla R, Adi PJ, et al. Novel 1, 4-dihydropyridines for L-type calcium channel as antagonists for cadmium toxicity. Sci Rep. 2017;7:45211

29. Teleb M, Zhang FX, Huang J, et al. Synthesis and biological evaluation of novel N3-substituted dihydropyrimidine derivatives as T-type calcium channel blockers and their efficacy as analgesics in mouse models of inflammatory pain. Bioorg Med Chem. 2017;25(6):1926-1938. 